\title{
Análisis químico del extracto peciolar para evaluar el estado nutricional en la vid
}

\author{
Paulina Pino(1), Rodrigo Callejas ${ }^{(1)}$, Bruno Razeto(2) y Gabino Reginato(1) \\ (1)Universidad de Chile (UCHILE), Centro Regional de Estudios Agronómicos Atacama, Rómulo J. Peña, no 231, Copiapó, Chile. \\ E-mail: paupino@uchile.cl (2)UCHILE, Facultad de Ciencias Agronómicas, Santa Rosa, o 11.315, La Pintana, Casilla 1.004, Santiago, Chile. \\ E-mail: rcalleja@uchile.cl, brazeto@uchile.cl, greginat@uchile.cl
}

\begin{abstract}
Resumen - El objetivo de este trabajo fue evaluar la relación existente entre la concentración de N, P y K del extracto peciolar, la lámina y la raíz, para determinar el estado nutricional de la vid. El ensayo se desarrolló entre diciembre de 2006 y febrero de 2007. Se validó la metodología de extracto peciolar para Vitis vinifera 'Red Globe', en plantas de un año de edad autoenraizadas, creciendo en macetas, a partir de tres ensayos de fertilización utilizando $\mathrm{N}, \mathrm{P}_{2} \mathrm{O}_{5}$ y $\mathrm{K}_{2} \mathrm{O}$. En el extracto peciolar, se evaluó nitrato, fosfato y potasio, y en láminas y raíces, nitrógeno, fósforo y potasio. El análisis del extracto peciolar fue más sensible que el de la lámina y la raíz para determinar el estatus de nitrato y nitrógeno. Mientras que el análisis de la lámina representa mejor la respuesta de la planta a la fertilización con fósforo y potasio. El extracto peciolar puede detectar antagonismos entre elementos en altas dosis de fertilización.
\end{abstract}

Términos para indexación: Vitis vinifera, análisis de savia, análisis de tejido, diagnóstico nutricional, 'Red Globe'.

\section{Petiole extract chemical analysis to evaluate nutritional status in grapevine}

\begin{abstract}
The objective of this work was to evaluate the relationship between N, P and K concentration in petiole extract, blades and roots, to determine the nutritional status in grapevine. The experiment was done between December 2006 and February 2007. Three fertilization trials with $\mathrm{N}, \mathrm{P}_{2} \mathrm{O}_{5}$ and $\mathrm{K}_{2} \mathrm{O}$ in one-year-old Vitis vinifera 'Red Globe' plants, grown in containers, were established. Nitrate, phosphate and potassium concentration were evaluated in the petiole extract, and nitrogen, phosphorus and potassium were determined in blades and roots. Petiole extract analysis was more sensitive than blade and root analysis to determine nitrate and nitrogen status. Meanwhile, blade analysis showed better plant response to fertilization with phosphorus and potassium. Petiole extract analysis can indicate the antagonism between nutrients under high doses of fertilization.
\end{abstract}

Index terms: Vitis vinifera, sap analysis, tissue analysis, nutritional diagnosis, 'Red Globe'.

\section{Introducción}

Para evaluar el estado nutricional de la vid (Vitis vinifera L.), tradicionalmente se ha empleado el análisis de pecíolo durante la floración y el de lámina en floración y en envero. No obstante, ambos tejidos han presentado inconsistencias entre potencial productivo y niveles nutricionales (Ruiz, 2000; Rombolá \& Sorrenti, 2006), transformándose en una limitante para mejorar la fertilización. Además, se han observado variaciones en la concentración de los nutrientes, según la variedad, edad del tejido, posición de la hoja, clima, época del año, momento de muestreo y presencia de residuos. Asimismo, faltan estándares para cada variedad, portainjerto, localidad y estado fenológico que faciliten la interpretación (Gil \& Pszczólkowski,
2007). Otro inconveniente de ambos análisis es el tiempo que requiere la preparación y análisis de la muestra, que puede tardar al menos diez días en la entrega de los resultados (Nagarajah, 1999; Alcántar et al., 2002), siendo que los sistemas de diagnóstico nutricional debieran ser rápidos, eficientes y permitir realizar correcciones inmediatas en el programa de fertilización (Gaviola \& Lipinski, 2004).

En la búsqueda de mejores indicadores del estado nutricional en la vid, se ha recurrido al análisis de otros tejidos, como raíces, sarmiento, yema floral, inflorescencia, raquis y bayas (Ruiz, 2000; Rombolá \& Sorrenti, 2006; Gil \& Pszczólkowski, 2007), de los cuales las raíces y sarmientos, en el período de receso invernal, detectan bien las diferencias en las reservas de N, P, K y carbohidratos, aunque las raíces muestran 
mayor sensibilidad (Ruiz, 2000). El análisis de yema floral en desarrollo también se ha usado para evaluar los niveles de reserva en el período de yema hinchada (Gil \& Pszczólkowski, 2007). Mientras que el análisis de raquis ha representado mejor que la lámina los efectos de la fertilización nitrogenada y potásica al suelo, y el análisis de inflorescencia ha detectado mejor que el de lámina las deficiencias de boro.

Adicionalmente, se ha desarrollado el análisis de extracto peciolar, que es más sensible en el diagnóstico de deficiencias y excesos en la nutrición de distintas especies hortícolas y frutales (Hidalgo et al., 2006), mostrando mayor amplitud que el análisis foliar en la concentración de los nutrientes (Villeneuve et al., 2002). Además, es una técnica más económica y rápida en relación a un análisis convencional (MacKown \& Weik, 2004; Capitán-Vallvey et al., 2005). Este análisis se ha utilizado para determinar el estado nutricional en olivo (Hidalgo et al., 2006) y en distintos cultivos hortícolas, tales como ajo (Gaviola \& Lipinski, 2004), tomate (Taber, 2001; Cadahía López, 2005), melón (Fontes et al., 2003), alcachofa (Rodrigo et al., 2005, 2006), brócoli (Villeneuve et al., 2002) y zapallo (Studstill et al., 2003). El principal compuesto que se ha analizado en el extracto peciolar es el nitrato (Matthäus \& Gysi, 2001; Taber, 2001; Villeneuve et al., 2002; MacKown \& Weik, 2004; Rodrigo et al., 2005, 2006) y, en menor medida, el potasio (Studstill et al., 2003; Hidalgo et al., 2006) y el fósforo (Hall \& Raynor, 2002).

Al igual que para otros frutales, existe escasa literatura acerca del uso del análisis de extracto peciolar en vid (Nagarajah, 1999; Cadahía López, 2005), por lo que su implementación debe ser evaluada. La validación se basa en establecer correlaciones entre la composición química del extracto peciolar con la fertilización empleada y, a su vez, con la composición química de la materia seca de las hojas (Studstill et al., 2003).

El objetivo de este trabajo fue evaluar la relación existente entre la concentración de N, P y K del extracto peciolar, la lámina y la raíz, para determinar el estado nutricional de la vid.

\section{Materiales y Métodos}

En el Campus Antumapu, de la Facultad de Ciencias Agronómicas de la Universidad de Chile, a 3334'12"S, $70^{\circ} 38^{\prime} 4^{\prime \prime} \mathrm{W}$, a $624 \mathrm{~m}$ de altitud, con clima templado cálido de veranos secos, según la clasificación Köppen, se establecieron plantas autoenraizadas de Vitis vinifera 'Red Globe', de un año de edad, cada una en macetas de 20 L, en sustrato de arena gruesa, cuya composición química era de $0,1 \mathrm{~g} \mathrm{~kg}^{-1}$ de $\mathrm{N}, 0,064 \mathrm{~g} \mathrm{~kg}^{-1}$ de $\mathrm{P}$ y $0,11 \mathrm{~g} \mathrm{~kg}^{-1}$ de $\mathrm{K}$, con conductividad eléctrica de 1,7 dS m ${ }^{-1}$, y $\mathrm{pH}$ de 7,93.

Previo a la plantación, se desinfectó el sustrato con tetratiocarbonato de sodio al 0,32\%. Después de siete días, se regó para lavar el sustrato y, a fines de primavera, en diciembre de 2006, se transplantaron las vides. El riego se realizó diariamente, tres veces al día, por 15 min cada vez, con un gotero de $4 \mathrm{~L}$ por hora por maceta. La composición química del agua de riego fue $10,4 \mathrm{mg} \mathrm{L}^{-1}$ de $\mathrm{N}, 4 \mathrm{mg} \mathrm{L}^{-1}$ de $\mathrm{P}$, 5,2 $\mathrm{mg} \mathrm{L}^{-1}$ de $\mathrm{K}$, con conductividad eléctrica de $1,25 \mathrm{dS} \mathrm{m}^{-1}, \mathrm{y} \mathrm{pH}$ de 7,79. Durante la temporada, se realizaron dos riegos de $45 \mathrm{~min}$, para evitar la acumulación de sales en la maceta.

Se realizaron tres ensayos de fertilización para probar, individualmente, dosis crecientes de $\mathrm{N}$ $\left(0,2,56,5,12,7,68\right.$ y 9,60 g por planta), $\mathrm{P}_{2} \mathrm{O}_{5}$ $\left(0,0,98,1,47,2,44\right.$ y 3,42 g por planta) y $\mathrm{K}_{2} \mathrm{O}$ $(0,2,30,4,61,6,91$ y $9,22 \mathrm{~g}$ por planta), en diseños completamente aleatorizados. Cada ensayo, a su vez, se fertilizó con una dosis fija de los elementos contrarios respecto del analizando, por ejemplo, el ensayo de nitrógeno se fertilizó con dosis fija de 2,44 $\mathrm{g} \mathrm{P}_{2} \mathrm{O}_{5}$ por planta y $6,91 \mathrm{~g} \mathrm{~K}_{2} \mathrm{O}$ por planta; para los otros ensayos, se usó 7,68 g N por planta. La fertilización se efectuó una vez por semana, durante enero y febrero del 2007, habiéndose dividido en ocho semanas la dosis mencionada de cada ensayo, para determinar el efecto de la fertilización de este período en la composición química del extracto peciolar. Los productos empleados fueron nitrato de amonio ( $33 \%$ de $\mathrm{N}$ ), ácido fosfórico $\left(62 \%\right.$ de $\left.\mathrm{P}_{2} \mathrm{O}_{5}\right)$ y sulfato de potasio $\left(51 \%\right.$ de $\left.\mathrm{K}_{2} \mathrm{O}\right)$. Terminada la fertilización, se colectaron las muestras foliares y de pecíolos a mediados de marzo. El muestreo se realizó entre las 9 y 13 h, después de un riego, y se colectaron todas las hojas y pecíolos de la planta. Posteriormente, en junio del 2007, se extrajeron las raíces de los tres ensayos más los sarmientos del ensayo de fósforo.

Elanálisis de lámina se realizó con cinco repeticiones, y el de extracto peciolar, raíz y sarmientos, con tres repeticiones. La unidad experimental fue constituida por dos plantas, para asegurar la obtención de $30 \mathrm{~g}$ de 
masa de pecíolos frescos, necesarios para realizar el análisis de extracto peciolar (Cadahía López, 2005). El extracto peciolar se obtuvo a través del trozado y congelado rápido de los pecíolos en éter etílico más hielo seco a -60 ó $-70^{\circ} \mathrm{C}$, para provocar la ruptura celular. Se pesaron $30 \mathrm{~g}$ de pecíolo congelado, se añadieron $30 \mathrm{~g}$ de agua destilada, y se trituraron en una licuadora por $1 \mathrm{~min}$, aproximadamente; luego se centrifugaron a $2.451 \mathrm{~g}$ por $5 \mathrm{~min}$; se filtró el líquido, con papel filtro Whatman no 41 , y se le realizó una desproteinización con alcohol etílico absoluto (desionizado con $1 \%$ de ácido acético reactivo), el cual se dejó reaccionar por ocho horas a $1^{\circ} \mathrm{C}$. La fracción proteica se separó por centrifugación y se purificó por lavado con alcohol etílico (del $75 \%$ en volumen con $1 \%$ de ácido acético reactivo). Luego, en columnas de fraccionamiento, se realizó la separación de la fracción aniónica y las moléculas no polares con el uso de resinas catiónicas fuertemente ácidas.

En el extracto peciolar, se evaluó la concentración de nitrato $\left(\mathrm{NO}_{3}^{-}\right)$, amonio $\left(\mathrm{NH}_{4}^{+}\right)$, fosfato $\left(\mathrm{PO}_{4}{ }^{3-}\right)$ y potasio $(\mathrm{K})$. En la lámina y raíces, se determinó la concentración de $\mathrm{N}$ total, $\mathrm{P}$ y K. Se analizó el Zn, en entrenudos de sarmientos del ensayo con dosis crecientes de $\mathrm{P}$, para evaluar antagonismos con los elementos estudiados en el extracto peciolar. En las hojas y raíces, se analizaron: el $\mathrm{N}$, por destilación y titulación manual; el $\mathrm{P}$, por colorimetría; y el $\mathrm{K}$, al igual que el $\mathrm{Zn}$ del sarmiento, por espectrofotometría de absorción atómica (Sadzawka et al., 2004).

Se realizó análisis de varianza, con la verificación previa de los supuestos sobre los términos del error. Se utilizó la prueba de Anderson-Darling, para verificar el supuesto de normalidad, y la prueba de Bartlett para la homogeneidad de varianzas. En caso de que los supuestos no se cumplieran, se transformaron las variables originales. La separación de las medias se realizó con la prueba de comparación múltiple de Tukey, al 5\% de probabilidad, cuando se detectaron diferencias.

Para determinar la relación entre la concentración de N, P y K del extracto peciolar, con la concentración en la lámina y la raíz, y con las dosis aplicadas de fertilizantes, se realizaron regresiones, que fueron sometidas a la prueba $\mathrm{F}$, al análisis de significancia para la pendiente y el punto de intersección con el eje, y a la verificación de las hipótesis del modelo. El coeficiente de correlación de Pearson (r) se evaluó con el estadístico t de Student.

\section{Resultados y Discusión}

Solo los análisis de extracto peciolar y de lámina detectaron diferencias entre las dosis crecientes de $\mathrm{N}$ (Figura 1). La variabilidad en la concentración de $\mathrm{NO}_{3}{ }^{-}$ del extracto peciolar, al aumentar la dosis de fertilización nitrogenada $(\mathrm{CV}=27,45 \%)$, probablemente se debe a los rápidos cambios que sufren su absorción y concentración a lo largo de la estación, asociados a períodos de mayor crecimiento, efectos hormonales, transporte del $\mathrm{N}$ en forma de aminoácidos o a la dificultad de mantener un abastecimiento constante de N (Matthäus \& Gysi, 2001). Al respecto, Nagarajah (1999) también observó las mismas variaciones en extracto peciolar de vid 'Thompson Seedless', a lo largo del día, en plena floración, y recomendó muestrear los pecíolos entre las 8 y 9:30 h para reducir el error. La evaluación de la concentración de $\mathrm{NO}_{3}{ }^{-}$en distintos estados fenológicos permite identificar el período donde es más estable y representativo de la fertilización (Alcántar et al., 2002). Además, parte del $\mathrm{N}$ del extracto peciolar puede ser orgánico, y su variación podría reflejar la dinámica de la nitrato reductasa en la raíz (Gil \& Pszczólkowski, 2007). La misma variabilidad del extracto peciolar ha sido reportada en naranjo, olivo, tomate, fresa (Cadahía López, 2005; Hidalgo et al., 2006) y brócoli (Villeneuve et al., 2002).

La fertilización explicó, en un mayor porcentaje, el contenido de $\mathrm{N}$ foliar $\left(\mathrm{R}^{2}=0,93\right)$, respecto del extracto peciolar $\left(\mathrm{R}_{\text {nitrato }}=0,74\right.$ y $\left.\mathrm{R}_{\text {amonio }}=0,46\right)$ y de la raíz $\left(\mathrm{R}^{2}=0,49\right)$. Los tres tejidos manifestaron una tendencia al aumento de la concentración de $\mathrm{N}$, a medida que se incrementó la fertilización (Figura 1). Sin embargo, la trayectoria del extracto peciolar es ascendente en toda su extensión, mientras que, en la lámina, el ascenso es hasta la dosis 5,12 g de N por planta, para descender de allí en adelante, lo que indicaría que la lámina solo diagnosticaría niveles de $\mathrm{N}$ normales, ya que las concentraciones observadas coinciden con el rango normal de concentración (19-25 $\mathrm{g} \mathrm{kg}^{-1}$ ) para láminas recientemente maduras, en verano. En cambio, el extracto peciolar representaría bien el estado nutricional del $\mathrm{N}$ en la vid, al aumentar el nivel de abastecimiento a niveles elevados.

Villeneuve et al. (2002) obtuvieron resultados semejantes, al comparar, bajo distintas dosis de $\mathrm{N}$, el contenido de clorofila foliar con la concentración de $\mathrm{NO}_{3}{ }^{-}$en extracto peciolar de brócoli. Conjuntamente, el coeficiente de determinación obtenido para $\mathrm{NO}_{3}{ }^{-}$, en 
el extracto peciolar, indica un buen grado de relación con la fertilización y se encuenta dentro del rango observado en otros estudios $\left(0,70 \leq \mathrm{R}^{2} \geq 0,97\right)$ (Alcántar et al., 2002; Fontes et al., 2003; Gaviola \& Lipinski, 2004). En consecuencia, el extracto peciolar aparece como el tejido más sensible para detectar diferencias entre distintos suministros de nitrógeno.

Si un tejido tiene un alto coeficiente de determinación con distintos órganos, para las concentraciones de un mismo nutriente, su grado de confiabilidad es mayor para emplearlo en el diagnóstico nutricional. Al respecto, el extracto peciolar solo evidenció una relación significativa con el análisis de lámina para el $\mathrm{NH}_{4}{ }^{+}\left(\mathrm{R}^{2}=0,69\right)$ (Figura 2). Probablemente, el alto coeficiente de variación del $\mathrm{NO}_{3}{ }^{-}$del extracto peciolar afectó su relación con la concentración de $\mathrm{N}$ foliar. Estos resultados concuerdan con los obtenidos por Alcántar et al. (2002), quienes relacionaron el $\mathrm{NO}_{3}{ }^{-}$de extracto celular de hojas con $\mathrm{N}$ foliar en ajo; pero, se contraponen a lo observado por Fontes et al. (2003), quienes encontraron una alta correlación entre el $\mathrm{NO}_{3}{ }^{-}$ de extracto peciolar con el $\mathrm{N}$ foliar en melón.

El extracto peciolary la lámina detectaron diferencias entre las dosis de fósforo, aunque solo la lámina manifestó una tendencia al alza con el incremento en la fertilización (Figura 1). La raíz mostró poca sensibilidad ante el aumento del $\mathrm{P}$, ya que solo mostró diferencias entre el testigo y las cuatro dosis de fertilización. Las variaciones encontradas en la concentración de $\mathrm{PO}_{4}{ }^{3-}$, en el extracto peciolar $(\mathrm{CV}=29,84 \%)$, probablemente,
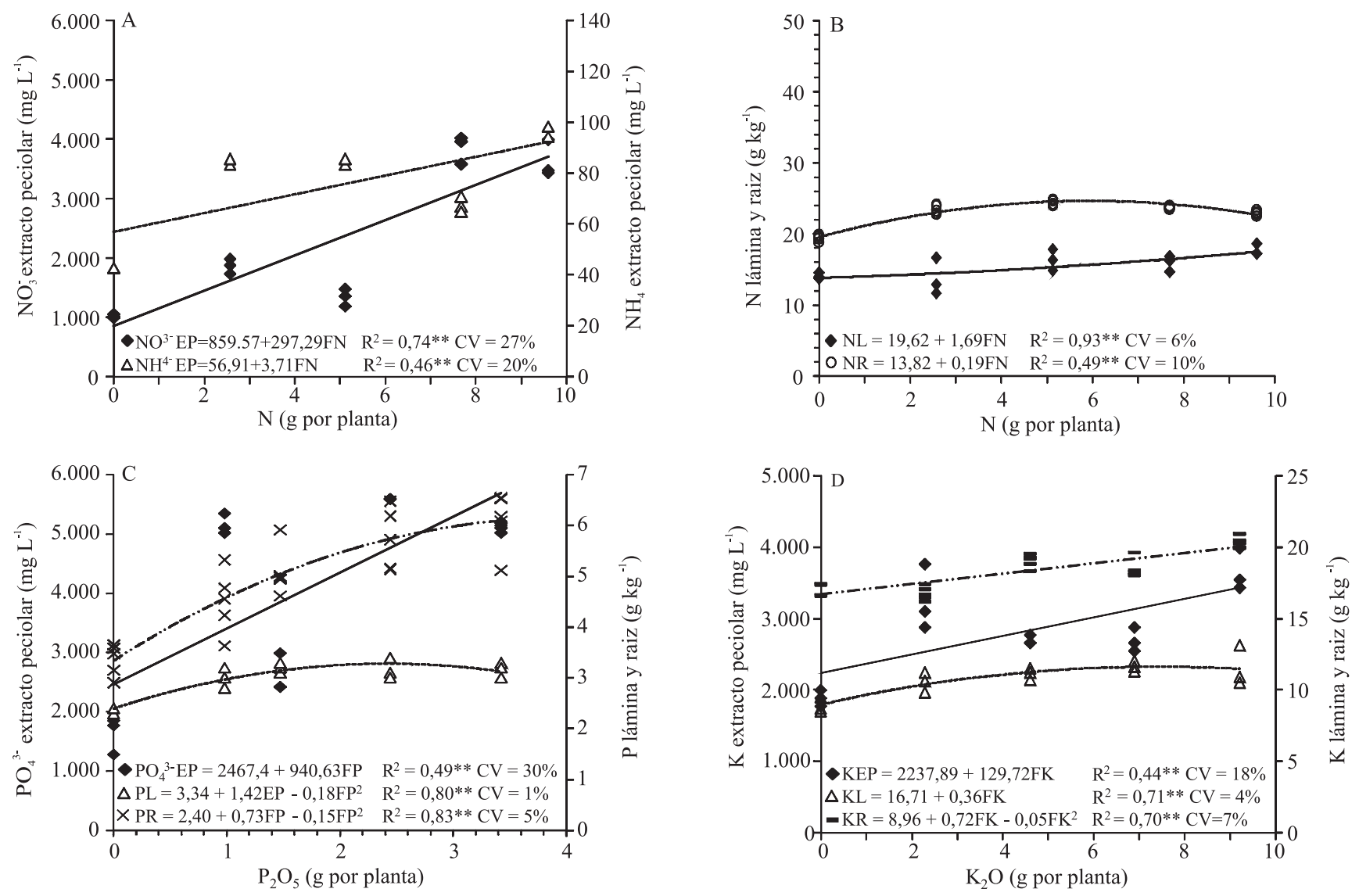

Figura 1. Concentración de nitrato y amonio en el extracto peciolar (A), y de nitrógeno, en la lámina y raíz (B), y su relación con las dosis crecientes de fertilización nitrogenada; $\mathrm{C}$, concentración de fosfato, en el extracto peciolar, y de fósforo, en la lámina y raíz, y su relación con las dosis crecientes de fertilización fosfatada; $\mathrm{D}$, concentración de potasio, en el extracto peciolar, lámina y raíz y su relación con las dosis crecientes de fertilización potásica. **Significativo al 1\%, por medio del estadístico t de Student. $\mathrm{NO}_{3}{ }^{-} \mathrm{EP}$, nitrato extracto peciolar; $\mathrm{NH}_{4}{ }^{+} \mathrm{EP}$, amonio extracto peciolar; $\mathrm{NL}$, nitrógeno lámina; $\mathrm{NR}$, nitrógeno raíz; $\mathrm{FN}$, fertilización nitrogenada; $\mathrm{PO}_{4}{ }^{3-\mathrm{EP}}$, fosfato extracto peciolar; PL, fósforo lámina; PR, fósforo raíz; FP, fertilización fosfatada; KEP, potasio extracto peciolar; KL, potasio lámina; KR, potasio raíz; FK, fertilización potásica. 
responden a los cambios que sufre la absorción por las raíces (Matthäus \& Gysy, 2001).

La fertilización fosfatada representó mejor al P raíz $\left(\mathrm{R}^{2}=0,83\right)$ (Figura 1); no obstante, su concentración solo fue ascendente hasta la dosis de $0,98 \mathrm{~g}$ de $\mathrm{P}_{2} \mathrm{O}_{5}$ por planta y, luego, se mantuvo. En cambio, el $\mathrm{P}$ de la lámina mostró un incremento, pero con una tasa de crecimiento descendente; y en el análisis de extracto peciolar, dicha curva fue ascendente en todo su recorrido. Estas trayectorias indicarían que la raíz es poco sensible para detectar excesos de $\mathrm{P}$ a mayores dosis de fertilización, mientras que la lámina muestra mayor sensibilidad, pero hasta un máximo. Por el contrario, la tendencia seguida por el extracto peciolar indica que se podrían detectar excesos en las concentraciones de $\mathrm{PO}_{4}{ }^{3-}$, al incrementar la dosis de fertilización; sin embargo, dicha tendencia es poco clara al fertilizar con $1,47 \mathrm{~g} \mathrm{P}_{2} \mathrm{O}_{5}$ por planta. En cualquier caso, todas las concentraciones de $\mathrm{PO}_{4}{ }^{3-}$ en el extracto peciolar fueron superiores a los valores de vid en estado de cuaje (267 mg L-1 de $\mathrm{PO}_{4}{ }^{3-}$ ) (Cadahía López, 2005), probablemente, por la alta concentración de $\mathrm{P}$ en el sustrato (equivalente a $311 \mathrm{~kg} \mathrm{ha}^{-1} \mathrm{de}$ P). Por su parte, la concentración de $\mathrm{P}$ foliar también se registró sobre los
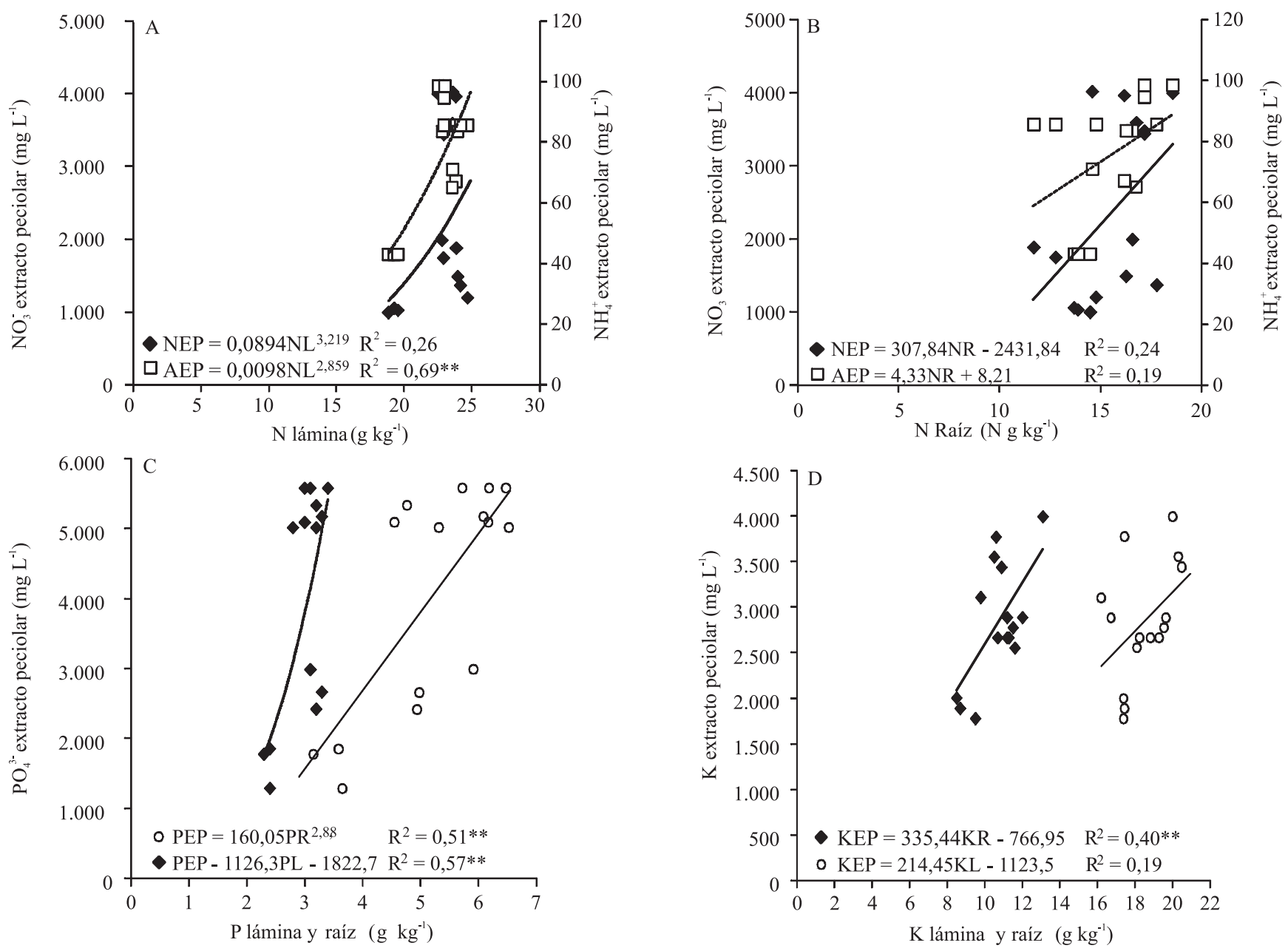

Figura 2. Relación entre la concentración de nitrato y amonio, en el extracto peciolar, y la concentración de nitrógeno en la lámina (A) y en la raíz (B), para el ensayo de dosis crecientes de fertilización nitrogenada; $\mathrm{C}$, relación entre la concentración de fosfato del extracto peciolar con la concentración de fósforo, en la hoja y en la raíz, para las dosis crecientes de fertilización fosfatada; D, relación entre la concentración de potasio del extracto peciolar con la concentración de potasio, en la hoja y en la raíz, para las dosis crecientes de fertilización potásica. **Significativo al 1\%, por medio del estadístico t de Student. NEP, nitrato extracto peciolar; AEP, amonio extracto peciolar; NL, nitrógeno lámina; NR, nitrógeno raíz; PEP, fosfato extracto peciolar; PL, fósforo lámina; $\mathrm{PR}$, fósforo raíz; KEP, potasio extracto peciolar; KL, potasio lámina; KR, potasio raíz. 
rangos normales de láminas recientemente maduras en verano (1,6-3,5 $\mathrm{g} \mathrm{kg}^{-1}$ ) (Ruiz, 2000). Adicionalmente, se detectó antagonismo entre la fertilización fosfatada y la concentración de $\mathrm{NO}_{3}{ }^{-}$del extracto peciolar $(\mathrm{r}=-0,88)$ y la concentración de zinc de los sarmientos $(\mathrm{r}=-0,60)$ (Figura 3), donde la lámina no mostró sensibilidad.

La concentración de $\mathrm{PO}_{4}{ }^{3-}$ en el extracto peciolar evidenció una relación significativa con aquella en la lámina $\left(\mathrm{R}^{2}=0,57\right)$ y en la raíz $\left(\mathrm{R}^{2}=0,51\right)$ (Figura 2), lo que concuerda con Nagarajah (1999), quien también obtuvo una estrecha relación entre el $\mathrm{PO}_{4}{ }^{3-}$ en el extracto peciolar y pecíolos de vides 'Thompson Seedless' en plena flor.

Los análisis de extracto peciolar y de lámina fueron más sensibles para detectar la respuesta a la fertilización potásica, dado que en la raíz solo se halló diferencias entre el testigo y las tres dosis de fertilización más altas.

La fertilización potásica explicó mejor la concentración de $\mathrm{K}$ foliar $\left(\mathrm{R}^{2}=0,71\right)$ y de la raíz $\left(\mathrm{R}^{2}=0,70\right)$ que el $\mathrm{K}$ del extracto peciolar $\left(\mathrm{R}^{2}=0,44\right)$ (Figura 1), y los tres tejidos mostraron una tendencia al aumento en la concentración de $\mathrm{K}$ con aumento de la dosis. Sin embargo, la lámina es la que mejor representa el estado nutricional de potasio, ya que presentó una relación lineal simple con la fertilización potásica, lo que indica una relación más fuerte entre la fertilización y la concentración de potasio en la lámina

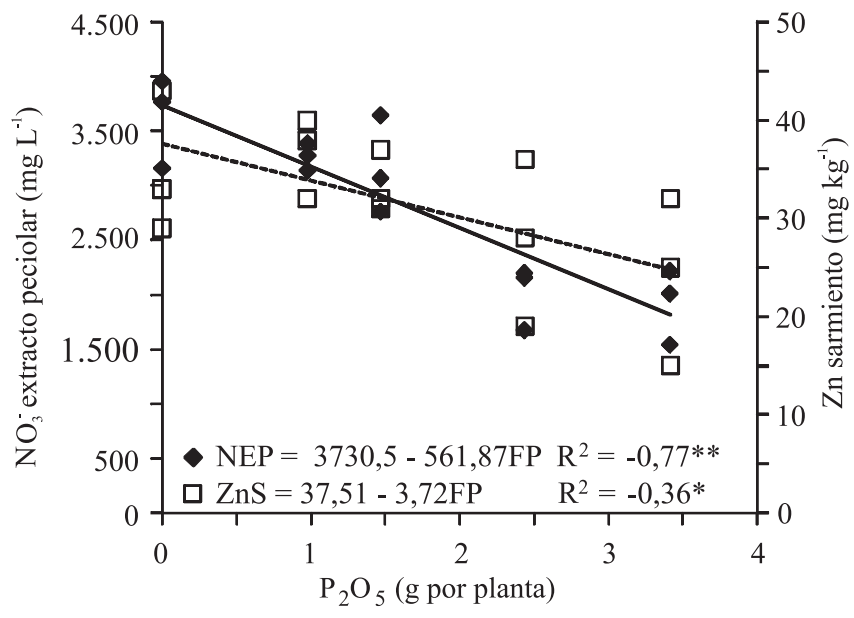

Figura 3. Concentración de nitrato, en el extracto peciolar, y de zinc, en el sarmiento, como función de las dosis crecientes de fertilización fosfatada. ** y *Significativo al 1 y al 5\%, por medio del estadístico t de Student. NEP, nitrato extracto peciolar; $\mathrm{ZnS}$, zinc sarmientos; FP, fertilización fosfatada. que la obtenida entre la raíz y el extracto peciolar con la fertilización. Estos resultados discrepan de los obtenidos por Nagarajah (1999), quien obtuvo un $\mathrm{R}^{2}=0,99$, al relacionar la concentración de $\mathrm{K}$ del extracto peciolar de vid 'Thompson Seedless' con la fertilización potásica; sin embargo, a diferencia de este estudio, el análisis lo realizó en plena flor y en plantas adultas. Hay que considerar que puede variar la concentración de $\mathrm{K}$ a lo largo de la estación de crecimiento, ya que la relación obtenida con la fertilización varía de acuerdo con el estado fenológico (Taber \& Lawson, 2007). La concentración de $\mathrm{K}$ en el extracto peciolar solo evidenció una relación significativa con el $\mathrm{K}$ en la raíz $(\mathrm{r}=0,63)$ (Figura 2).

\section{Conclusiones}

1. El análisis de extracto peciolar es más sensible que el análisis de lámina y de raíz para determinar el estado de nitrógeno en la vid, y detecta respuesta de las concentraciones de nitrato y amonio hasta altas dosis de fertilización nitrogenada.

2. El análisis de lámina es más preciso que el análisis de extracto peciolar y de raíz para evaluar el estado de fósforo y potasio.

3. El análisis de extracto peciolar presenta una elevada sensibilidad para detectar antagonismos entre elementos, cuando las dosis de fertilización son altas.

4. La concentración en el extracto peciolar presenta una fuerte asociación con la concentración en lámina para amonio y fosfato, y con la concentración en raíz para fosfato y potasio.

\section{Referencias}

ALCÁNTAR, G.; SANDOVAL, M.; CASTELLANOS, J.; MÉNDEZ, F.; SÁNCHEZ, P.; RODRÍGUEZ, M.N. Diagnostic methods to evaluate nutrient status of garlic, onion, and broccoli. Communications in Soil Science and Plant Analysis, v.33, p.2585-2598, 2002.

CADAHÍA LÓPEZ, H. (Coord.). Fertirrigación: cultivos hortícolas, frutales y ornamentales. 3.ed. Madrid: Mundi-Prensa, 2005. 683p.

CAPITÁN-VALLVEY, L.F.; ARROYO-GUERRERI, E.; FERNÁNDEZ-RAMOS, M.D.; SANTOYO-GONZALEZ, F. Disposable receptor-based optical sensor for nitrate. Analytical Chemistry, v.77, p.4459-4466, 2005.

FONTES, P.C.; COELHO, E.; CARDOSO, A.A. Petiole sap nitrate and leaf nitrogen critical values in melon plants grown in unheated 
greenhouse and field conditions. Journal of Plant Nutrition, v.26, p.1403-1411, 2003.

GAVIOLA, S.; LIPINSKI, V.M. Evaluación de rendimiento y nitratos en ajo cv. Nieve INTA con riego por goteo. Agricultura Técnica, v.64, p.172-181, 2004.

GIL, G.F.; PSZCZÓLKOWSKI, P. Viticultura: fundamentos para optimizar producción y calidad. Santiago: Universidad Católica de Chile, 2007. 535p.

HALL, R.; RAYNOR, K. Nutrient analysis for the nursery industry. Combined Proceedings of the International Plant Propagators' Society, v.52, p.126-129, 2002.

HIDALGO, J.C.; FRUTOS, I.; NIETO, J.; CADAHÍA, C.; VEGA, C.; PASTOR, M. Fertilización potásica en olivar de regadío de la variedad Picual. Influencia sobre el contenido de potasio en hoja, savia y frutos. Fruticultura Profesional, v.161, p.107-111, 2006.

MACKOWN, C.; WEIK, J.C. Comparison of laboratory and quick-test methods for forage nitrate. Crop Science, v.44, p.218-226, 2004.

MATTHÄUS, D.; GYSI, C. Plant-sap analysis in vegetables: a tool to decide on nitrogen top dressing. Acta Horticulturae, v.563, p.93-102, 2001.

NAGARAJAH, S. A petiole sap test for nitrate and potassium in Sultana grapevines. Australian Journal of Grape and Wine Research, v.5, p.56-60, 1999.

RODRIGO, M.C.; GINESTAR, J.; BOIX, M.; RAMOS, C. Evaluation of rapid methods for nitrate plant sap analysis of globe artichoke grown in sand culture. Acta Horticulturae, v.697, p.393-397, 2005.
RODRIGO, M.C.; GINESTAR, J.; RAMOS, C. Evaluation of rapid methods for nitrate sap analysis in artichoke. Acta Horticulturae, v.700, p.237-240, 2006.

ROMBOLÁ, A.D.; SORRENTI, G. Fertilización y diagnosis del estado nutricional de las especies frutales. Fruticultura Profesional, v.161, p.5-18, 2006.

RUIZ S., R. Dinámica nutricional en cinco parrones de diferente productividad del valle central regado de Chile. Agricultura Técnica, v.60, p.379-398, 2000.

SADZAWKA R., A.; GREZ Z., R.; CARRASCO R., M.A.; MORA G., M. de la L. Métodos de análisis de tejidos vegetales. Santiago: Sociedad Chilena de la Ciencia del Suelo, 2004. 53p.

STUDSTILL, D.W.; SIMONNE, E.H.; HUTCHINSON, C.M.; HOCHMUTH, R.; DUKES, M.; DAVIS, W. Petiole sap testing sampling procedures for monitoring pumpkin nutritional status. Communications in Soil Science and Plant Analysis, v.34, p.2355-2362, 2003.

TABER, H. A petiole sap nitrate sufficiency values for fresh market tomato production. Journal of Plant Nutrition, v.24, p.945-959, 2001.

TABER, H.; LAWSON, V. Use of diluted tomato petiole sap for potassium measurement with the cardy electrode meter. Communications in Soil Science and Plant Analysis, v.38, p.713-718, 2007.

VILLENEUVE, S.; COULOMBE, J.; BELEC, C. ; TREMBLAY, N. A comparison of sap nitrate test and chlorophyll meter for nitrogen status diagnosis in broccoli (Brassica oleracea L. spp. italica). Acta Horticulturae, v.571, p.171-177, 2002.

Recibido en 19 de abril de 2011 e aprobado en 13 de diciembre de 2011 